\title{
Vorkommnisse mit Medizinprodukten melden - Sicherheit verbessern
}

\author{
Zahlen zur Fehlerkultur bei Produktproblemen in der Medizintechnik zeigen, dass \\ Spitäler und andere professionelle Anwender deutlich zu wenige schwerwiegende \\ Vorkommnisse mit Medizinprodukten an Swissmedic melden.
}

Andreas Schlegel

Korrespondenz:

Dr. phil. nat. Andreas Schlegel Swissmedic, Schweizerisches Heilmittelinstitut

Abteilung Medizinprodukte Hallerstrasse 7

CH-3000 Bern 9

Tel. 0313227701

Fax 0313227646

andreas.schlegel(at)

swissmedic.ch

\begin{abstract}
Anwender melden zu wenig
Hersteller haben in der Schweiz zwischen Januar 2002 und Dezember 2010 knapp 3000 Meldungen über schwerwiegende Vorkommnisse mit Medizinprodukten bei Swissmedic eingereicht. Anwender hingegen nur rund 750. Die Anzahl der Anwendermeldungen liegt damit weit hinter der Anzahl von Herstellermeldungen zurück. Bei den Meldungen von Herstellern ist seit 2005 ein ununterbrochener Anstieg zu beobachten (Abb. 1). Die Meldungen von Schweizer Anwendern haben dagegen seit 2003 stagniert.

Bei Herstellermeldungen stammen rund $60 \%$ der Meldungen aus der deutschen Schweiz. Die übrigen Meldungen entsprechend dem Bevölkerungsanteil aus den französischen und italienischen Landesteilen. Bei Anwendermeldungen hingegen kommen lediglich 51\% der Meldungen aus der deutschen, dafür aber 34\% aus der französischen und ein der Bevölkerung entsprechender Anteil aus der italienischen Schweiz. Anwender aus der französischen Schweiz melden damit signifikant häufiger als ihre Kollegen in deutschsprachigen Regionen.
\end{abstract}

\section{Abbildung 1}

Anzahl Vorkommnisse pro Jahr, die von Anwendern bzw. Herstellern an Swissmedic gemeldet wurden.

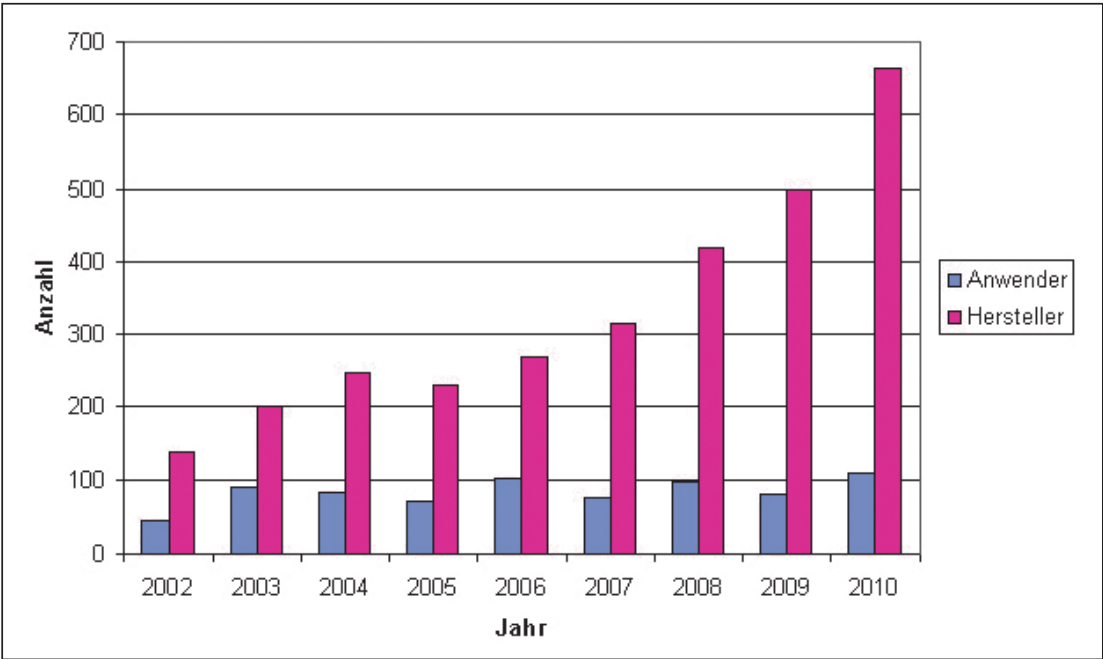

\section{Signaler les incidents avec des dispositifs médicaux pour améliorer la sécurité}

Les hôpitaux et autres professionnels de la santé qui utilisent des dispositifs médicaux ne signalent que trop rarement les incidents critiques à Swissmedic. C'est ce qu'illustrent les derniers chiffres en matière de gestion des erreurs lors de problèmes liés à des dispositifs médicaux. Comparé aux fabricants, les utilisateurs n'annoncent qu'un incident sur quatre. Pour les dispositifs médicaux implantables, ce taux est encore plus bas. Pourtant, si les utilisateurs signalaient les problèmes, ils profiteraient de mesures de sécurité accrues et obtiendraient de meilleurs produits.

\section{Anwendermeldungen bewirken vermehrt Sicherheitsmassnahmen am Produkt}

Hersteller führten, wenn sie selbst gemeldet hatten, in $81 \%$ der Fälle keine Sicherheitsmassnahme durch, weil sie keinen Fehler am Produkt feststellten oder das gemeldete Problem nicht bestätigen konnten. In $14 \%$ der Fälle führten die herstellenden Firmen nach Herstellermeldungen eine Sicherheitsmassnahme durch, z.B. einen Rückruf oder einen Warnhinweis (Abb. 2). Wenn Vorkommnisse von Anwendern gemeldet wurden, führten die Hersteller hingegen in $25 \%$ der Fälle eine Sicherheitsmassnahme durch. Damit ist bei Anwendermeldungen der Anteil Fälle signifikant höher, bei denen zugunsten der Allgemeinheit Sicherheitsmassnahmen ergriffen werden.

\section{Anwender vernachlässigen Meldungen zu implantierbaren Produkten}

Die prozentualen Anteile verschiedener Produktkategorien (gemäss GMDN [1]) sind bei Anwender- und Herstellermeldungen unterschiedlich (Abb. 3). Im- 


\section{Abbildung 2}

Anteil der gemeldeten Vorkommnisse, die eine Sicherheitsmassnahme (z. B. Rückruf oder Warnhinweis) bzw. keine Massnahme zur Folge hatten. (n. i.: Ende 2010 noch nicht abgeschlossene Untersuchungen).

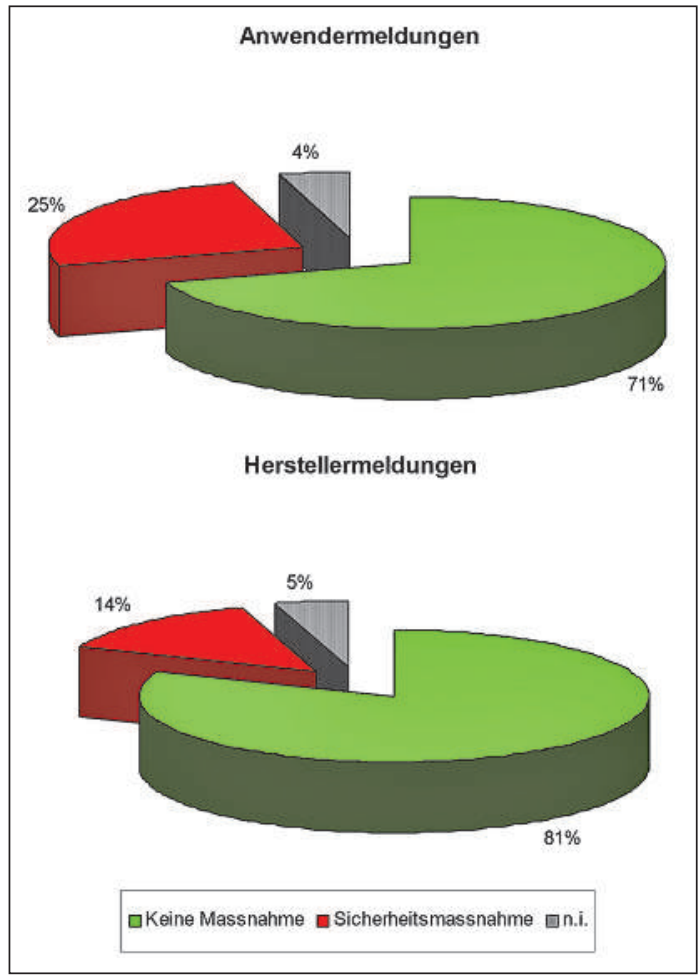

plantierbare Produkte sind bei Anwendermeldungen signifikant untervertreten, besonders krass die aktiven implantierbaren Produkte (z. B. Herzschrittmacher, Defibrillatoren, Elektroden) mit einem Anteil von bloss $3 \%$ gegenüber $22 \%$ bei Herstellermeldungen. Umgekehrt ist der Anteil an Spital-Hardware, elektromechanischen Produkten und Einwegproduk-

\section{Abbildung 3}

Anteil Meldungen pro Produktkategorie. Produktkategorien wurden gestützt auf die internationale Medizinproduktenomenklatur (GMDN) wie folgt definiert: 1. Spital-Hardware, 2. Elektromechanische Produkte, 3. Einwegprodukte, 4. Anästhesie- und Respirationsprodukte, 5. Nichtaktive implantierbare Produkte, 6. Aktive implantierbare Produkte, 7. In-vitro-Diagnostika (IVD) und 8. Andere GMDN-Kategorien (n.i.: nicht codierte Produkte).

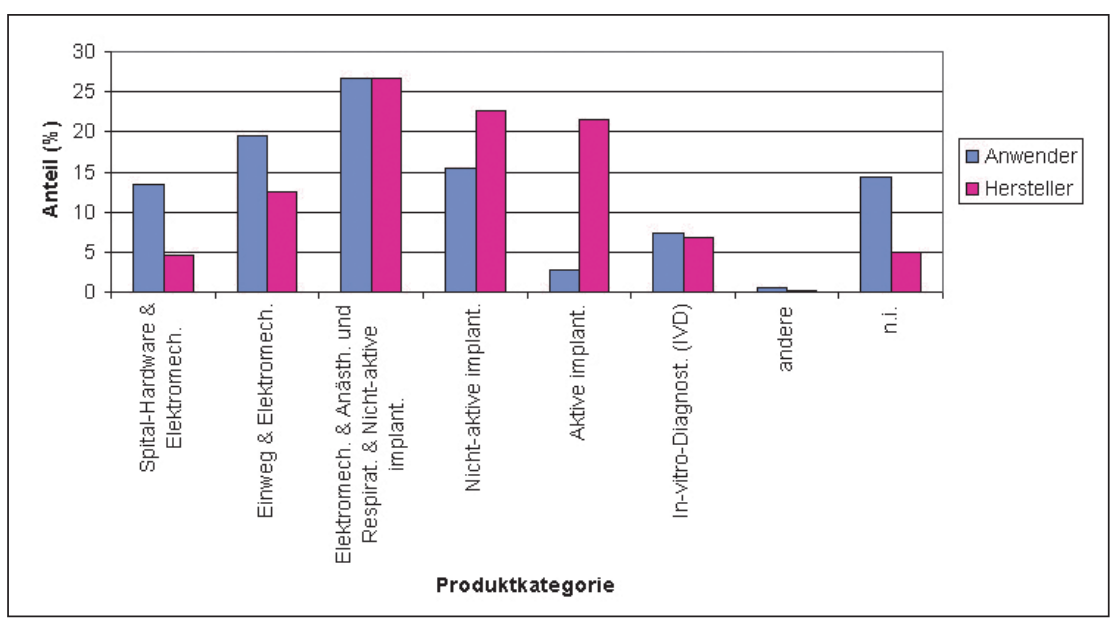

ten bei Anwendermeldungen höher als bei Herstellermeldungen.

\section{Diskussion und Ausblick}

Die Zahlen in diesem Bericht zeigen, dass Spitäler und andere Anwender mit ihren Vorkommnismeldungen wesentlich zur Verbesserung der Sicherheit von Medizinprodukten beitragen. Professionelle Anwender melden aber zu wenig und nützen das Poten-

\section{Schwerwiegende Vorkommnisse mit Medizinprodukten müssen gemäss Art. 15 Medizinprodukte- verordnung an Swissmedic gemeldet werden.}

tial zur Verbesserung der Sicherheit daher nur suboptimal. Insbesondere Institutionen, die implantierbare Medizinprodukte verwenden, hätten die Möglichkeit, mehr Vorkommnisse zu melden. Es ist davon auszugehen, dass die Hersteller mehrheitlich von Anwendern über stattgefundene Vorkommnisse informiert werden, weshalb die Anwender von den meisten Vorkommnissen wohl Kenntnis haben. Es sollte nicht sein, dass Anwender schwerwiegende Vorkommnisse nur selektiv und bei ausgewählten Produkten an die Behörde melden, die für die Produktsicherheit zuständig ist.

\section{Gesetzliche Basis}

Professionelle Anwender und Hersteller müssen schwerwiegende Vorkommnisse mit Medizinprodukten gemäss Art. 15 Medizinprodukteverordnung an Swissmedic melden [2]. Diese Meldepflicht besteht für Hersteller seit 1996, und seit 2002 für professionelle Anwender. Swissmedic stellt den Anwendern und Herstellern im Internet Meldeformulare und eine umfangreiche Dokumentation zur Verfügung [3]. Swissmedic lädt Kontaktpersonen der Schweizer Spitäler seit 2002 regelmässig zu Tagungen ein, wo das Erstellen solcher Meldungen geschult wird. Diverse Publikationen informieren über die Meldepflicht $[4,5]$.

\section{Literatur}

1 Global Medical Device Nomenclature. GMDN (www.gmdnagency.com).

2 Medizinprodukteverordnung, SR 812.213 (www.admin.ch/ch/d/sr/c812_213.html).

3 www.swissmedic.ch/md.asp

4 Schlegel A. Das Medizinprodukte-Vigilancesystem am Beispiel von Blutzuckermesssystemen. Schweiz Ärztezeitung. 2007;88(41):1711-4.

5 Schlegel A. Materio-Vigilance Meldungen durch Labors. Pipette. 2005;1:20-2. 